\title{
58
}

\section{Software Development Environments to Support Model Consistency}

\author{
V.E.Veraart and S.L. Wright \\ Computer Science Programme, Murdoch University, Perth, Western Australia.
}

\begin{abstract}
A major problem affecting software quality and software development productivity is the use of incorrect and inconsistent models of the problem solution domains during the software development process. Our solution is firstly to make the methods and models more precise by defining their semantics, and secondly to ensure that partially overlapping models are semantically and syntactically consistent over their alias components. Our approach is expose the operations a tool performs on its model to other tools via semantic events. We maintain tool independence by using a co-ordinator to translate semantic events in one model into appropriate semantic events on alias components in other models.
\end{abstract}

Keyword codes: D.2.6; D.2.1; D.2.2.

Keywords:Programming Environments; Requirements/Specifications; Tools and Techniques.

\section{INTRODUCTION - METHODS AND TOOLS}

The use of methods for modelling various aspects of a proposed system has been an accepted part of systems analysis and design for many years. As software development proceeds through the lifecycle the nature of the models changes as the information contained in individual functional, data and time models is aggregated into models that become more concrete in respect to the final system. Development converges to the "final solution" models. These may be represented as source code fragments, database queries, library functions, etc. As convergence proceeds many of the inconsistencies are usually discovered, often after considerable economic investment in the project. We believe that the creation of consistent, complementary models enhances the capability, correctness, and therefore quality, of the final, implementation. Our particular area of interest is ir the application of analysis and high-level design methods to produce complementary models for the early phases of the software development lifecycle (SDLC).

\section{MODEL CONSISTENCY}

The deliverables of the analysis and design phases of the SDLC are usually a set of models representing particular views of the system. These models are not a deliverable as far as the client is concerned, but the information they contain is a deliverable to some downstream activity in the software manufacturing process. (If this is not the case, then there is no justification for building them.) As such, they form an important part of the infrastructure required for software development: and as with any infrastructure component, contribute indirectly to the quality of the final product.

\subsection{Alias components}

If we consider that each of the models represents aview of the problem domain or system under development, then by definition these concurrent models possess a degree of commonality. This commonality arises from the alias components that occur in the models. We define an alias 
component to be an entity (in its broadest sense of an item, place, event, role, policy etc) that appears in a number of method-models but with different semantics and representations in each model. The analyst naturally identifies items as alias components as part of the model-development process.

\subsection{Model evolution}

Software development proceeds in an iterative manner. Once the initial models have been created, we often need to update domain information. Typically, this is done from within a particular tool. This tool can maintain its internal model consistency. However, in order for inter-model consistency to be preserved any changes we make to the model components must be propagated to all relevant alias components in other models. If consistent change propagation is not incorporated into the development process, we can have a set of models that are no longer consistent in reflecting the state of our knowledge of the problem domain or system [6].

\section{SEMANTIC TOOL INTEGRATION}

Our work seeks to identify some of the reasons that tool integration is important, in terms of the needs of the users of an environment, and to propose an integration mechanism that meets those needs. We propose that the solution to the problem of model consistency is to integrate method-tools with respect to the commonality between the models they manipulate.

\subsection{Syntactic and semantic specification of method-models}

All method-models have a syntax, a notation that describes the valid components of the model and its structure. This syntax can be expressed as a concrete syntax using either graphical components or textual lexemes. For graphical models the syntax is most often expressed as a set of "rules" describing the shape and placement of their components, using narrative text.

Within a model some combinations of components, although syntactically valid may be semantically meaningless Model semantics involves the rules, relating to the types of components, that govern the consistency between a model and its "owner" ie method. Thus flow, transform, store, etc, are the 'data types' of the DFD method and there is a set of rules that constrains how they can be operated upon, eg combination. We use a type system that allows us to introduce the notion of semantic checks to eliminate many obvious semantic errors

Once we move beyond the simple creation operations, the definition of the method semantics for many operations is often left to the individual tool implementer; there are no ISO or ANSI standards in this area. Clearly, the semantics of any operation should be clearly specified, unambiguously, in terms of its effect on its models. Failure to achieve thiswithin any single method-tool makes integration with any other tool impossible.

To achieve consistency between models that are the product of different method-tools, we must be able to map operations on the components of one method-model onto alias components represented in other models. Since the differences between models are likely to be both syntactic and semantic (depending on the semantic-gap between tools) the mapping operation must be able to operate at both syntactic and semantic levels. We define models having a high degree of similarity of form and expression as exhibiting a small semantic gap. Semantic-closeness is generally low for method-tools that are used in the earlier phases of the SDLC, ie analysis and high-level design.

A commonly proposed solution to the semantic-gap problem is to create unified operation sets that can operate on all method-models of the environment. However, reducing all the semantic operations to a common, canonical set has obvious problems.

\section{A COOPERATIVE APPROACH TO TOOL INTEGRATION}

Our alternative approach is to express the required "consistency" operations in terms of each tool's own operation set. This allows independent tools to be inserted into, and removed from, an integrated 
environment without requiring modifications to any other tool in the environment. This is achieved by specifying each method's operation-set as a formal, concise semantic definition. Once the set of alias components for the methods in the environment has been identified, we determine which operations of their operations have the potential to produce model inconsistencies. Finally we define the operation-mappings that are necessary across tools whose models are affected by these operations.

Most analysis tools are not directly integrable due to the semantic gap between them in terms of their operations and data components. We group these tools into acluster, and all the tools are linked to a coordinator. The coordinator is a programmable component that gives us flexiblity in defining an environment's toolset. Our tools are required to publicise their behaviour, this establishing a basis for a communication method between tools. When a tool cooperates with other tools in its cluster it informs them of any relevant actions on its own model by issuingsemantic events. A semantic event is an operation on a method-model, by its tool, that is syntactically and semantically valid for the model and causes a change in the state of the model. Each tool defines a set of semantic events that are equivalent to operations on its models.

The cluster coordinator handles mapping of outgoing tool-events into semantically consistent ingoing events by table lookup, simple translation or by invocation of specified computation fragments. By introducing both a coordinator-cluster mechanism, and semantic events, we can ensure that tools in the environment are totally independent of one another. The coordination component is a fully programmable part of the system. For example, when a tool is modified, or a new tool added to the environment, other tools are not directly affected by any new semantic events. Rather, the coordinator is modified to translate any new events into events that are meaningful in terms of other existing tools.

For very simple semantic operations automatic consistency control may be possible, however at this stage we envisage that user intervention will be necessary and a tool's response to events may be to inform the user of the need for changes to the model and to identify the affected components. To handle consistency control automatically one solution is to include an expert-system as an additional component in the environment, whose rules encapsulate "consistency policies".

\section{TECHNICAL ISSUES}

To take advantage of the event-coordination strategy, tools will usually be written specifically for the environment. Method tools are implemented as small, cohesive micro-tools, and assembled around a cluster coordinator This allows for a high degree of reuse, between tools, of the common components that are found in many tools eg as object drawing functions, cut-and-paste editing functions, etc.

To integrate a tool into the environment it requires:

- an executable component that implements each tool's functions and can be directly integrated into a cluster

- a place to store their model data structures - a file store, or similar mechanism

- a set of method-model data structure definitions that can be installed into the store

- an external interface definition, that is a tool's events; a method-tool must have a well defined set of operations in terms of syntax (notation) and semantics (model manipulation) a set of fragments to handle the events as defined by each tool's external interface definition. the ability to execute in an identical manner whether they are run in stand-alone mode or in a cluster.

The interface descriptions are used to decide how tools can be connected, and to validate the connections in terms of correct data type matching, etc. Architectural requirements for the environment include:

- an event handling mechanism

- ability to run tools and coordinators as separately executing processes, or threads 
- the means to communicate data between tools

- facilities for setting up communication paths between tools, and/or perform broadcasts.

Many current operating systems, for example UNIX, provide the basic technical mechanisms that enable us to build a prototype. We are currently building a prototype to test our concepts, and highlight inadequacies, using $\mathrm{C}++$, UNIX, and Glish [4] to provide the inter-process communication.

\section{RELATED WORK}

Very little work on integration is done from an environment user's perspective, ie of what use is integration to them. Brinkkemper adopts a similar approach to our work with alias components. He uses meta-data modelling to derive method-companionship[1] that is used in defining editor transfer charts, in the context of switching between editors, on the execution of certain editing operations concerned with common components. We apply meta-modelling at the level of events on models of data structures rather than to diagrammatic representations. A number of recent papers have appeared in the literature concerning integration of structured methods such as entity-modelling and data flow diagramming with formal methods [5] [3] [2]. In general, these deal with uni-directional translation, rather than method integration which we consider to be bi-directional. Our approach allows updates to be made in any of the clustered tools, and to be notified to other tools with models containing common components.

\section{CONCLUSION}

We have proposed that one method for improving the quality of software is by exerting some control over the models created during the development process. The control takes the form of requiring consistency between the models. Ensuring that inconsistencies are noted and corrected as soon as possible ensures that flaws relating to missing or ambiguous information do not become apparent in the latter stages on the development process.

Implementing integration at the tool level means that the actual methods remain unchanged. To achieve model-consistency via tool-integration we identify the alias components of the method models and ensure that operations on these components, executed within the method-tool, are made available to any other tools, sharing a common responsibility for the component, in a manner that is semantically consistent for each tool. We achieve this by clustering the tools about a coordination tool that acts as a semantic event translator and despatcher. The tools communicate via events that are comprehensible within each tool in terms of its own model semantics.

In our cooperative approach there is no requirement for tools to agree on common data types, structures and/or message formats, thus it prevents loss of independence and flexibility. The definitions for a tool's data structures, as well as its control over instantiated data structures, are encapsulated within the tool itself. This differs from the common approach of data being maintained in a common repository where access is only available via the repository manager. From the perspective of its user the tool behaves as any normal independent tool, and can be used in a totally stand-alone capacity. Cooperative tools can easily be reused and can be attached to other groups of tools, providing a very flexible integration arrangement. These benefits are achieved through each tool working at a high-level of abstraction (manipulating its method-models) and exposing its operations, at that model level, to other tools using semantic events.

An extended version of this paper is available, via FTP on ftp.cs.murdoch.edu.au as Murdoch University Computer Science Technical Report CS/94/05. 


\section{REFERENCES}

1. Brinkkemper, S., deLange, M., Looman, R., van der Steen, F.H.G.C., 'On the derivation method-companionship by meta-modelling', Soft. Eng. Notes, (15) 1, January 1990, pp 49-58.

2. Misic, V., Velasevic, D., Lazarevic, B., 'Formal specification of a data dictionary for an extende ER data model', Computer Journal, (35) 6, 1992, pp 611-622.

3. Polack, Fiona, 'Integrating formal notations and systems analysis: using entity relation-ship diagrams", Software Engineering Journal, September 1992, pp 363-371.

4. Paxson, Vern., Saltmarsh, Chris., 'Glish: A user-level software bus for loosely-couplec distribution systems', Proceedings of 1993 Winter USENIX Technical Conference, 1993.

5. Semmens, L.T., France, R.B., Docker, T.W.G., 'Integrated structured analysis and form specification techniques', Computer Journal, (35) 6, 1992, pp 600-610.

6. Veraart, V.E., Wright, S.L., 'Consistent models enhance software qualityProceedings of SQM94,

Computational Mechanics Institute, Edinburgh, July 94, pp 159-174. 